\title{
Berry's phase in cavity QED: Proposal for observing an effect of field quantization
}

\author{
A. Carollo, ${ }^{1,2}$ M. França Santos, ${ }^{1}$ and V. Vedral ${ }^{1, *}$ \\ ${ }^{1}$ Optics Section, The Blackett Laboratory, Imperial College, London SW7 2BZ, United Kingdom \\ ${ }^{2}$ INFM, Unità di Ricerca di Milano, via Bramante 65, I-26013 Crema (CR), Italy
}

(Received 7 May 2002; published 16 June 2003)

\begin{abstract}
We propose a feasible experiment to investigate quantum effects in geometric phases, arising when a classical source drives not a single quantum system, but two interacting ones. In particular, we show how to observe a signature of the quantization of the electromagnetic field through a vacuum effect in Berry's phase. To do so, we describe the interaction of an atom and a quantized cavity mode altogether driven by an external quasiclassical field. We also analyze the semiclassical limit recovering the usual Berry's phase results.
\end{abstract}

DOI: 10.1103/PhysRevA.67.063804

PACS number(s): 42.50.Ct, 03.65.Vf

Geometric phases, or Berry's phases, are well known in classical electromagnetism and quantum mechanics since the early works of Pancharatnam [1] and Berry [2]. Their origin relies on the geometric nature of state spaces and has been studied in many different systems, such as spins, polarized light, and atomic physics [3]. Recent works have explored their application in interferometry and quantum computation $[4,5]$.

Earlier works on the subject suggest how to observe these phases in single quantum systems adiabatically driven by external classical devices or sources, where, by classical, we mean any system whose state does not change considerably during the interaction time; an intense magnetic field interacting with a spin $\frac{1}{2}$ or a birefringent medium interacting with polarized light [6-11].

A more recent work explores new geometrical effects arising from the interaction of two independent quantized systems [12]. In particular, the cyclic evolution of a twolevel system interacting with two independent quantized harmonic oscillators is analyzed, especially when the state of the harmonic oscillator is the vacuum. It is shown that the quantization of the modes introduces deviations from the predictions of the correspondent semiclassical model, where the two-level system interacts with a classical polarized field.

Interactions of this nature, between two-level systems and quantized harmonic oscillators, have been the subject of many experimental investigations in the last two decades, specially in the cavity QED domain [13-16]. Many of these experiments test the quantum nature of light itself. In this paper, we propose a different experiment in cavity QED that allows one to observe and measure the novel geometric effects described in Ref. [12].

In the above, the theoretical model considered is a resonant Jaynes-Cummings interaction, coupling a two-level system of Bohr frequency $\nu$ and a quantized mode of creation and annihilation operators $\hat{a}$ and $\hat{a}^{\dagger}$ :

$$
H_{0}=\nu \hat{a}^{\dagger} \hat{a}+\nu \hat{b}^{\dagger} \hat{b}+\nu \hat{\sigma}_{22}+\lambda\left(\hat{\sigma}_{21} \hat{a}+\hat{\sigma}_{12} \hat{a}^{\dagger}\right),
$$

where $\hat{\sigma}_{i j}=|i\rangle\langle j|(i, j=1,2)$, with $|1\rangle$ and $|2\rangle$ ground and exited states of the two-level system, respectively. A second

\footnotetext{
*Electronic address: v.vedral@ic.ac.uk
}

mode with creation and annihilation operators $\hat{b}$ and $\hat{b}^{\dagger}$ and frequency $\nu$ initially is not interacting with the particle nor with the first mode.

The initial Hamiltonian is then adiabatically transformed by linearly combining the two modes according to the following:

$$
\begin{aligned}
H(\theta, \phi)= & \nu \hat{\sigma}_{22}+\nu\left(\hat{a}^{\dagger} \hat{a}+\hat{b}^{\dagger} \hat{b}\right)+\lambda\left[\left(\cos \frac{\theta}{2} \hat{a} e^{-i(\phi / 2)}\right.\right. \\
& \left.\left.+\sin \frac{\theta}{2} \hat{b} e^{i(\phi / 2)}\right) \hat{\sigma}_{21}+\text { H.c. }\right],
\end{aligned}
$$

where $\theta$ and $\phi$ are two parameters, which can be changed externally. Formally, this transformation of the modes can be represented by a point of coordinates $\theta$ and $\phi$ on a sphere, the Poincaré sphere (see Fig. 1).

It is shown that by changing adiabatically the initial Hamiltonian $H_{0}$ according to this transformation, a system

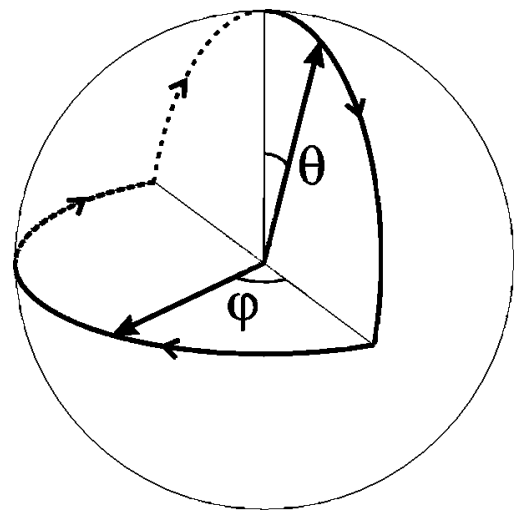

FIG. 1. A closed loop described by the polarization vector of the external driving field. The (classical) geometric phase generated in this way is equal to one-half of the solid angle enclosed by the path (equal to $\pi$ in this figure). Note that the same picture can be used to represent the evolution of a quantum two-level system whose state space has the same structure as a two-dimensional sphere (known as the Bloch sphere). In our paper, we combine these two pictures with the quantization of the field to produce and measure a new phase effect. The evolution of the joint atom and the quantized-field state can no longer be represented in this simple way. 
initially prepared in an eigenstate $\left|\psi_{n, m}^{ \pm}\right\rangle=1 / \sqrt{2}(|e, n\rangle$ $\pm|g, n+1\rangle)|m\rangle$ of $H_{0}$ (where $|n\rangle$ and $|m\rangle$ are the Fock states of modes $\hat{a}$ and $\hat{b}$, respectively) acquires the geometric phase given by

$$
\chi_{n, m}=\frac{1}{2} \gamma\left(n-m+\frac{1}{2}\right)
$$

where $\gamma$ is the solid angle enclosed in the path traversed by the parameters $\theta$ and $\phi$ on the Poincaré sphere (see Fig. 1). Note that both $\left|\psi_{n, m}^{+}\right\rangle$and $\left|\psi_{n, m}^{-}\right\rangle$, for fixed values of $n$ and $m$, acquire the same geometric phase $\chi_{n, m}$, which implies that the states $|e, n, m\rangle$ and $|g, n+1, m\rangle$ do, too. The most remarkable case is obtained with initial state $|e, 0,0\rangle$ $=1 / \sqrt{2}\left(\left|\psi_{0,0}^{+}\right\rangle+\left|\psi_{0,0}^{-}\right\rangle\right)$. In this case, the adiabatic evolution determines a Berry's phase

$$
\chi_{\text {zero }}=\frac{\gamma}{4}
$$

This result presents two interesting aspects, both related to the quantized nature of the harmonic oscillators. First, the two-level system acquires a phase even for initially empty modes, a typical signature of Rabi oscillations. Second, and more important, this phase is half the value obtained in the equivalent semiclassical model, as shown in Ref. [12], allowing for yet another method to clearly distinguish between classical and quantized harmonic oscillators.

In order to propose an experiment to observe this effect, we use a cavity QED setup to engineer the parameter dependent Hamiltonian (2). Our scheme relies on two Raman interactions in the $\Lambda$ configuration, as shown in Fig. 2(b). Transitions $1 \leftrightarrow 3$ and $1 \leftrightarrow 4$ are coupled by means of dispersive Jaynes-Cummings interactions [17] to two orthogonally polarized cavity modes, with respective coupling constants $g_{+}$and $g_{-}$( + for the right and - for the left polarizations). Transitions $2 \leftrightarrow 3$ and $2 \leftrightarrow 4$ are coupled by externally injected quasiclassical fields, with respective Rabi frequencies $\Omega_{+}$and $\Omega_{-}$. The Hamiltonian for the whole system in the interaction picture is given by

$$
\begin{aligned}
H_{\text {int }}= & \hbar\left(g_{+} \hat{\sigma}_{31} \hat{a}_{+}+g_{-} \hat{\sigma}_{41} \hat{a}_{-}+\Omega_{+} \hat{\sigma}_{32}+\Omega_{-} \hat{\sigma}_{32}\right) e^{-i \delta t} \\
& + \text { H.c., }
\end{aligned}
$$

where $\hat{a}_{+}\left(\hat{a}_{-}\right)$denotes the annihilation operator of the right (left) circularly polarized quantized mode, and we assume the same detuning $\delta$ for each transition, as shown in Fig. 2.

Due to the dispersive nature of the interactions, the two upper electronic levels of the atom act only as virtual states allowing for the two-photon coupling between levels $|1\rangle$ and $|2\rangle$. Following the same procedure described in Ref. [18], for large detuning, i.e., $\delta \gg g, \Omega$, we adiabatically eliminated levels $|3\rangle$ and $|4\rangle$. The whole system, then, evolves according to the effective Hamiltonian a

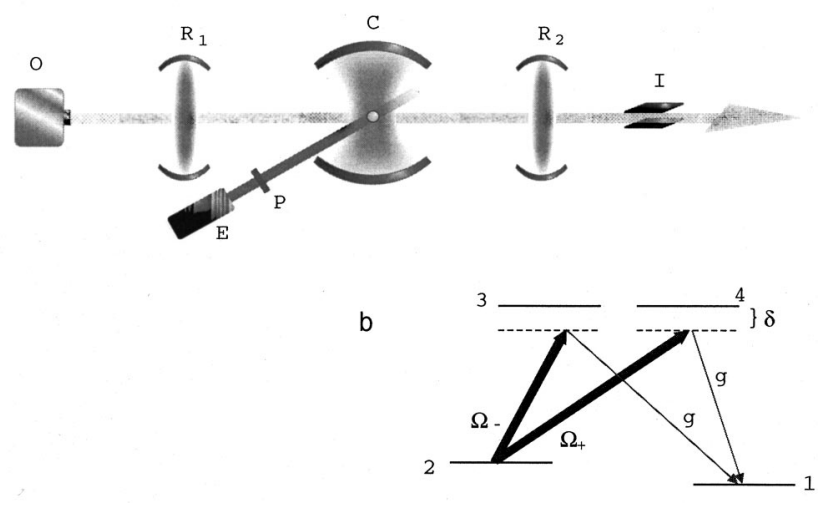

FIG. 2. The experimental proposal. The Rydberg atoms cross cavity $C$ one at a time; the cavity mode is initially prepared in some weak coherent state and a circularly polarized intense field $\vec{E}(t)$ drives the atom-cavity mode system. The atomic levels are shown in (b). For large detunings $\delta \gg g, \Omega$, the two lower levels interact effectively through a two-photon process; for simplicity, we consider $g=\Omega$, where $\Omega_{+}=\cos (\theta / 2) e^{i \phi / 2} \Omega$ and $\Omega_{-}$ $=\sin (\theta / 2) e^{-i \phi / 2} \Omega$; in $R 1$, the atoms are prepared in symmetric quantum superpositions of levels 1 and 2 , and subsequently perform an integral number of Rabi flips inside $C$. During this interaction in $C$, the polarization of the external field is slowly rotated so as to complete a closed loop in the Poincaré sphere (as in Fig. 1); the atoms are finally rotated back in $R 2$, and detected in $\mathrm{I}$.

$$
\begin{aligned}
H_{e f f}= & \hbar \frac{\Omega^{2}}{\delta} \hat{\sigma}_{22}+\hbar \frac{g^{2}}{\delta}\left(\hat{a}_{+}^{\dagger} \hat{a}_{+}+\hat{a}_{-}^{\dagger} \hat{a}_{-}\right) \hat{\sigma}_{11} \\
& +\lambda\left[\left(\cos \frac{\theta}{2} e^{i(\phi / 2)} \hat{a}_{+}+\sin \frac{\theta}{2} e^{-i(\phi / 2)} \hat{a}_{-}\right) \hat{\sigma}_{21}+\text { H.c. }\right],
\end{aligned}
$$

In Eq. (6), we express the polarization of the external field so that $\Omega_{+}=\cos (\theta / 2) e^{i \phi / 2} \Omega$ and $\Omega_{-}=\sin (\theta / 2) e^{-i \phi / 2} \Omega$. In this way, its free parameters $\theta$ and $\phi$ coincide with the ones described in (2). Note that, by changing the external field polarization, we can control the effective coupling between transition $|1\rangle \rightarrow|2\rangle$ and the cavity modes $\hat{a}_{+}$and $\hat{a}_{-}$. For example, if $\theta=0\left(\Omega_{-}=0\right)$, the atom is coupled only to mode $\hat{a}_{+}$. For general values of $\theta$ and $\phi$, this effective interaction will reproduce exactly the one described by Hamiltonian (2). Therefore, by rotating the polarization of the injected quasiclassical field, we can perform the cyclic evolution in the parameters space described in the theoretical model. We are also assuming, for the sake of simplicity, that the atom couples with the same strength to each polarization, both with the classical and the quantized modes $g_{+}=g_{-}$ $=g=\Omega$ and we define $\lambda=g \Omega / \delta$ as the effective coupling constant of the whole system.

Once the proper interaction is engineered, the geometric phase shift acquired by the atom-cavity modes composite system can be observed through the usual Ramsey interferometric method (Fig. 2) [13]. The atom is prepared in the first 
Ramsey zone and put into a symmetric superposition of levels $|1\rangle$ and $|2\rangle$, while mode $a_{+}$is prepared in some arbitrary state and mode $a_{-}$is prepared in the vacuum. The initial polarization of field $\vec{E}(t)$ is chosen so that only mode $a_{+}$ interacts with the atom. The external field is turned on and the whole system is allowed to interact inside the cavity for a time $\tau \gg 1 / \lambda$, during which the polarization of the external field is rotated at a much slower rate compared to $\lambda$ until it is back to its original value. After that, the atom undergoes a $\pi / 2$ rotation in the second Ramsey zone and is detected by ionization.

If the initial state of mode $a_{+}$is also the vacuum, then $H_{\text {eff }}$ is resonant (for $\Omega=g$ ) and the probability to detect the atom in level $|2\rangle$, after an integral number of Rabi flips inside the cavity and the $\pi / 2$ pulse of the second Ramsey zone, is given by $P_{2}=(1-\cos \gamma / 4) / 2$. This corresponds to a $\gamma / 4$ geometric phase shift, which is a surprising effect, comparing to the usual $\gamma / 2$ classical result. Note that the JaynesCummings interaction is particularly appropriated to observe this effect. The atom-cavity mode system splits into doublets except for a lonely ground state, which does not acquire any phase at all. Therefore, this lowest energy level works as an intrinsic reference frame for the measurement of the global geometric phase acquired by the remaining dressed states. This structure is only possible due to the existence of a vacuum state of the cavity mode, which becomes clear when the experiment is repeated with mode $a_{+}$initially prepared in a coherent state $|\alpha\rangle$. Then, the probability to measure the atom in state $|2\rangle$ for the same $\gamma$ is given by

$$
2 P_{2}=\left(1-e^{-|\alpha|^{2}}\right)(1-\cos \gamma / 2)+e^{-|\alpha|^{2}}(1-\cos \gamma / 4) .
$$

From this equation it is clear that only for $|\alpha\rangle=0$, we have the surprising $\gamma / 4$ ratio. As the intensity of the initial coherent state increases, and the role of that lonely reference frame is reduced, the observed phase shift goes to the well expected $\gamma / 2$ semiclassical limit. In this calculation, we assume a slightly different setup in which the dynamical effects can be eliminated, following the results described in Ref. [19].

In Fig. 3(a), we show the phase shift in the probability $P_{2}$ to find the atom in level $|2\rangle$ for an induced geometric phase $\gamma=\pi$ due to the rotation of the polarization of the driving field. In Fig. 3(b), we show $P_{2}$ as a function of different initial coherent states in the cavity field, for a fixed interaction time $\tau=10 / \lambda$ and the same phase shift $\gamma=\pi$.

Typical values for the one-photon vacuum Rabi frequency are $g / 2 \pi \simeq 50 \mathrm{kHz}[13,20]$. Choosing the same coupling for the classically driven transition $\Omega / 2 \pi \simeq 50 \mathrm{kHz}$, and a detuning $\delta=3 \Omega, \lambda / 2 \pi \simeq 15 \mathrm{kHz}$, which means that the atom-field system can perform approximately ten complete Rabi cycles during an effective interaction time of $0.6 \mathrm{~ms}$. Times of interaction of this order are achievable in microwave cavities for atomic velocities of the order of $10 \mathrm{~m} / \mathrm{s}$ and are within typical decaying times $(1 \mathrm{~ms})$ for the cavities used in Ref. [13]. According to our calculations, in these experimental conditions, the nonideal adiabatic rotation of the polarization produces errors of the order of $5 \%$ in $P_{2}$, which are smaller
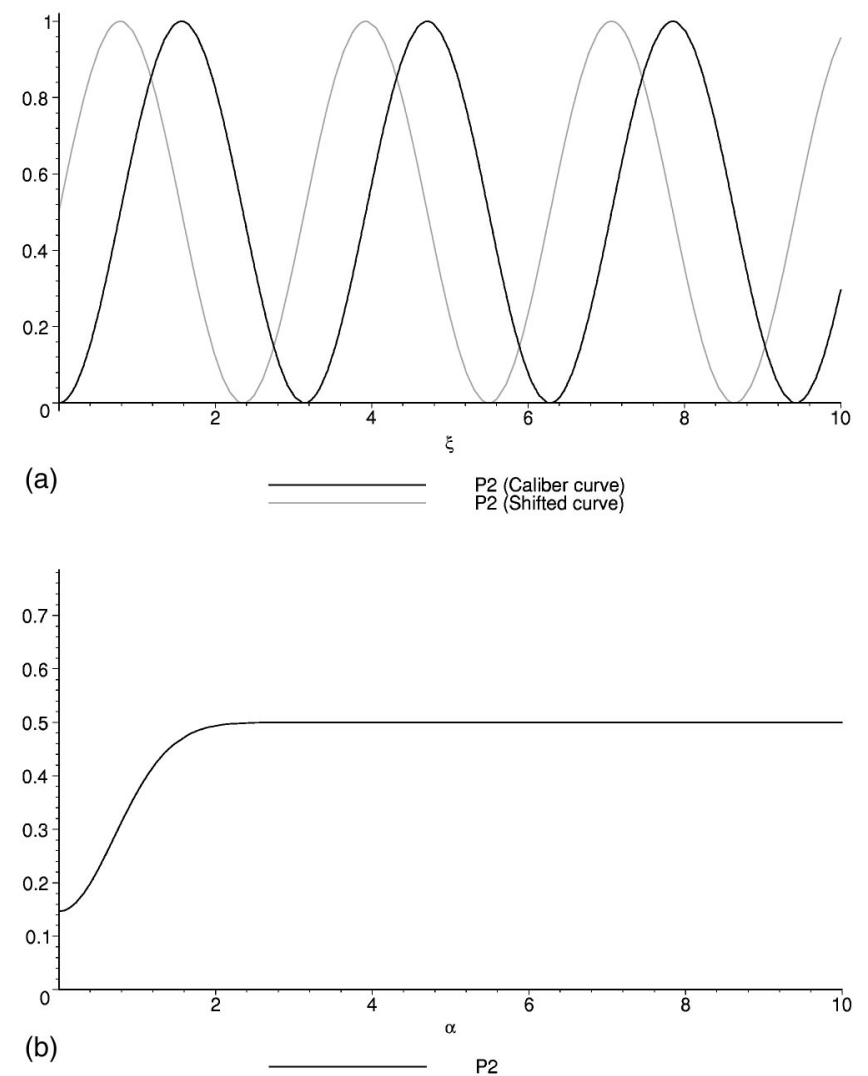

FIG. 3. Experimental prediction. (a) Probability $P_{2}$ to measure the atom in state $|2\rangle$, for a fixed number of Rabi flips inside the cavity and different relative phases $\xi$ between $R 1$ and $R 2$; the black curve is the caliber curve for the Ramsey interferometry and the gray curve represents the situation in which a geometric phase shift of $\pi / 4$ is induced in the system, according to the proposed experiment. This phase shift corresponds to the solid angle $\gamma=\pi$ described in Fig. 1. Part (b) represents the probability $P_{2}$ for different coherent states prepared in the cavity, as a function of the amplitude $\alpha$ of the coherent state (for each value of $\alpha$, we assume to set the interferometer such that $P_{2}=0$ in absence of geometric shift). We see that for the vacuum states (i.e., a coherent state of amplitude 0 ) the shift would be equal to $\pi / 4$ (i.e., $P_{2}=1 / 2[1-\cos (\pi / 4)]$ ), and it would increase with the coherent-state amplitude converging to the expected (semiclassical) value of $\pi / 2$ (i.e., $P_{2}=1 / 2[1-\cos (\pi / 2)]$ ). Note that the convergence is very fast, and for amplitudes of two and higher the semiclassical result is in a very good agreement with the fully quantized calculation.

than the ones produced by other sources, such as detectors inefficiency and cavity field decay.

In this paper, we have proposed an experimental setup to observe and measure the geometric behavior of two interacting quantized systems. We analyze a cavity QED experiment in which an externally injected field drives the interaction between a flying atom and two quantized cavity modes. The joint system acquires a geometric phase whose value is half of a two-level ( $\operatorname{spin} \frac{1}{2}$ ) system driven classically. We note in passing that this behavior is reminiscent of a particle with spin- $\frac{1}{4}$ fractional statistics. The model hereby described could also be easily translated into trapped ions systems in the Lamb-Dicke regime, where orthogonal vibration modes 
of the ions would play the role of the quantized harmonic oscillators.

In this work, we also clarify the physical origin of the model described in Ref. [12]. In particular, we show how to simulate the rotation of the polarization of quantized modes by engineering an effective Hamiltonian that produces the same final geometrical effect on the dressed atom-field states.

Our experimental proposal also reinforces the versatility and power of setups combining a quantized cavity mode manipulated by an externally injected quasiclassical field. The precise control of the properties of a quasiclassical external field allows one to engineer different atom-cavity modes in- teractions. In particular, in this paper, we show how to explore these interactions to investigate intrinsic geometric characteristics of a two-level system dressed by orthogonal harmonic oscillators. Similar setups could be used to further understand these geometrical properties as, for example, their effect on the collapses and revivals of atomic population in the Jaynes-Cummings model [21]. Furthermore, the addition of cavity mode damping to the model could also help understanding the geometric behavior of mixed states.

This research was supported by EPSRC, Hewlett-Packard, Elsag spa, and the EU. A.C. acknowledges the support of "Fondazione Angelo della Riccia." M.F.S. acknowledges the support of CNPq.
[1] S. Pancharatnam, Proc.-Indian Acad. Sci., Sect. A 44, 247 (1957).

[2] M.V. Berry, Proc. R. Soc. London, Ser. A 392, 45 (1984).

[3] Geometric Phases in Physics, edited by A. Shapere and F. Wilczek (World Scientific, Singapore, 1989).

[4] J.A. Jones, V. Vedral, A. Ekert, and G. Castagnoli, Nature (London) 403, 869 (1999).

[5] G. Falci, R. Fazio, G.M. Palma, J. Siewert, and V. Vedral, Nature (London) 407, 355 (2000).

[6] A. Tomita and R.Y. Chiao, Phys. Rev. Lett. 57, 937 (1986).

[7] D. Suter, G.C. Chingas, R.A. Harris, and A. Pines, Mol. Phys. 61, 1327 (1987).

[8] R. Bhandari and J. Samuel, Phys. Rev. Lett. 60, 1211 (1988).

[9] D. Suter, K.T. Mueller, and A. Pines, Phys. Rev. Lett. 60, 1218 (1988).

[10] P.G. Kwiat and R.Y. Chiao, Phys. Rev. Lett. 66, 588 (1991).

[11] C.L. Webb et al., Phys. Rev. A 60, R1783 (1999).
[12] I. Fuentes-Guridi, A. Carollo, S. Bose, and V. Vedral, Phys. Rev. Lett. 89, 220404 (2002).

[13] G. Nogues et al., Nature (London) 400, 239 (1999).

[14] J.M. Raimond, M. Brune, and S. Haroche, Rev. Mod. Phys. 73, 565 (2001).

[15] B.T.H. Varcoe, S. Brattke, M. Weidinger, and H. Walther, Nature (London) 403, 743 (2000).

[16] S. Brattke, B.T.H. Varcoe, and H. Walther, Phys. Rev. Lett. 86, 3534 (2001).

[17] B.W. Shore and P.L. Knight, J. Mod. Opt. 40, 1195 (1993).

[18] M.F. Santos, E. Solano, and R.L. de Matos Filho, Phys. Rev. Lett. 87, 093601 (2001).

[19] G. Morigi, E. Solano, B. Englert, and H. Walther, Phys. Rev. A 65, 040102(R) (2002).

[20] A. Rauschenbeutel et al., Phys. Rev. A 64, 050301(R) (2001).

[21] J.H. Eberly, N.B. Narozhny, and J.J. Sanchez-Mondragon, Phys. Rev. Lett. 44, 1323 (1980). 